\title{
Obesity - Can Allium fistulosum L. be a remedy?
}

Rownak Jahan ${ }^{1}$, Alok Paul ${ }^{2}$, Khoshnur Jannat ${ }^{1}$, Anamul Hasan ${ }^{1}$, Tohmina Afroze Bondhon ${ }^{1}$, Mohammed Rahmatullah ${ }^{1 *}$ ${ }^{1}$ Department of Biotechnology \& Genetic Engineering,University of Development Alternative, 3/F Satmasjid Road, Lalmatia, Dhaka-1207, Bangladesh.

${ }^{2}$ School of Pharmacy and Pharmacology, University of Tasmania, Hobart, Tasmania, Australia.

*Corresponding Author: Mohammed Rahmatullah, Dean, Faculty of Life Sciences, University of Development Alternative, 3/F Satmasjid Road, Lalmatia, Dhaha-1207, Bangladesh.

Received Date: 30 June 2021 | Accepted Date: 23 July 2021 | Published Date: 31 July 2021

Citation: R Jahan, A Paul, K Jannat, A Hasan, M Rahmatullah, et al. (2021) Obesity - Can Allium fistulosum L. be a remedy?. J. Endocrinology and Disorders. 5(4): DOI:10.31579/2640-1045/078

Copyright: (c) 2021 Mohammed Rahmatullah. This is an open-access article distributed under the terms of the Creative Commons Attribution License, which permits unrestricted use, distribution, and reproduction in any medium, provided the original author and source are credited. 2021

\begin{abstract}
Obesity is one of the major problems facing human beings at present. Because of the consumption of refined sugar and foods rich in carbohydrates, as well as adoption of a more sedentary lifestyle (often by necessity as is the case with most office workers), people in all countries are increasingly getting obese, which in turn is leading to other disorders like diabetes and cardiovascular complications. Existing conventional drugs have adverse sideeffects, which is the major cause for scientists to look towards the plant kingdom for discovery of newer drugs with less or no side-effects. The Allium genera of plants are widely known for their medicinal properties, in particular Allium cepa (onion) and Allium sativum (garlic), whose beneficial actions include anti-obesity effects. In this mini review, we examine a lesser known plant of the Allium genera, namely Allium fistulosum L. and review its anti-obesity potential. The conclusion of the present study is that the plant contains phytochemicals like ferulic acid and quercetin, which possess anti-obesity mechanisms of action thus rendering the plant to be of potential use against obesity.
\end{abstract}

Key words: obesity; adipose tissue; ferulic acid; quercetin; allium fistulosum

\section{Introduction}

Obesity and overweight has been defined by World Health Organization (WHO) "as abnormal or excessive fat accumulation that may impair health". The WHO factsheet of June 9, 2021 puts forth some statistics on obesity, which indicates a tripling of obese persons in the world since 1975; among people 18 years of age or older, more than 1.9 billion adults were overweight in 2016 with 650 million obese persons; these numbers in 2016 indicated that $39 \%$ of the world population were overweight and $13 \%$ suffered from obesity; 39 million children were obese or overweight in 2020; and over 340 million children and adolescents of the world aged 5-19 years were obese or overweight in 2016. The ironic part of the situation is that in countries with the most obese or overweight people, more people die of being obese than from malnutrition [1].

According to WHO "body mass index (BMI) is a simple index of weightfor-height that is commonly used to classify overweight and obesity in adults. It is defined as a person's weight in kilograms divided by the square of his height in meters $\left(\mathrm{kg} / \mathrm{m}^{2}\right)$ ". Also, according to WHO, a BMI of 25 or more in adults is classified as overweight, and a BMI of 30 or more is classified as obesity [1]. The rapid rise in the numbers of obese and overweight persons is generally attributed to increased intake of foods that are high in fat and refined sugar, and a more sedentary lifestyle consisting of less outdoor activity and more armchair office and home activities. Foods that are high in fat and refined sugar may be sugary drinks, pizzas, white bread, fruit juices, sweetened breakfast cereals, pastries, cookies or cakes and fried, grilled or boiled food [https://www.healthline.com/nutrition/20-foods-to-avoid-like-theplague\#TOC_TITLE_HDR_7].

As per WHO, the risk of non-communicable diseases increases with BMI. These diseases include cardiovascular diseases [2], diabetes [3], musculoskeletal disorders (like osteoarthritis) [4], and cancers including endometrial, breast, ovarian, prostate, liver, gallbladder, kidney, and colon cancers [5]. Increased waist circumference of more than 40 inches in men ( 35 inches in women) is known as visceral adiposity and can be a cause for danger even though BMI is at normal level.

Pro-inflammatory cytokines are closely linked with obesity. A crosssectional study comprising of 117 obese patients with a body mass index of 30 or above and 83 non-obese volunteer persons found that general obesity was closely linked with various interleukins (IL) like ILs 5, 10, 12 , and 13 along with interferon- $\gamma$ (IFN- $\gamma$ ) and tumor necrosis factor- $\alpha$ (TNF- $\alpha$ ). General obesity patients with a low degree of physical activity had elevated levels of ILs 4, 10 and 13 [6]. The results are strongly suggestive that obesity cannot be simply explained away as the difference between energy intake and energy expenditures, rather more complex factors are at play. Adipocytes are not just fat reservoirs, but can secrete a wide range of bioactive peptides and proteins, which collectively are known as adipokines and can play a part in obesity [7]. 
Adepokinomes, which constitute the secretome of fat cells, release not only fatty acids and prostaglandins, but also proteins involved in the alternative complement system (adipsin, acylation-stimulating protein), insulin resistance, lipid metabolism (retinol-binding protein, cholesteryl ester transfer protein), vascular hemostasis (plasminogen activator inhibitor-1 or PAI-1), blood pressure regulation (angiotensinogen), glucose homeostasis (adiponectin), and angiogenesis (vascular endothelial growth factor or VEGF) [7]. Other proinflammatory cytokines that are released from adepokinomes include tumor necrosis factor- $\alpha$ (TNF- $\alpha$ ), IL-1 $\beta$, ILs 6,8 , and 10 , transforming growth factor- $\beta$, plasminogen activator inhibitor-1, haptoglobin, and serum amyloid A [8, 9]. Production of these factors and proteins are increased in obesity [7] and have been hypothesized to lead to a chronic low-grade inflammation leading to insulin resistance and metabolic syndrome like disorders. Obesity markers include IL-6, TNF- $\alpha$, C-reactive protein, and haptoglobin $[10,11]$. In fact, the hypothesis has reversed from obesity being the factor behind secretion of proinflammatory cytokines to obesity being an inflammatory disease that is obesity is an effect of proinflammatory cytokine(s) production in the body as a result of other factor(s) $[11,12]$.

Breast feeding has been shown to reduce obesity. Breast milk is rich in long-chain polyunsaturated fatty acids (LCPUFAs), which inhibit the production of proinflammatory cytokines and enhance the number of insulin receptors in various tissues [13, 14]. As such lack of LCPUFAs in the body/diet can lead to obesity. In rodent models, n3-LCPUFAs have been found to have a protective effect against high fat diet-induced insulin resistance. The protective action of n3-LCPUFAs has been attributed to the prevention of decrease of phosphatidyl inositol 3' (PI3) kinase activity and the prevention of the depletion of glucose transporter 4 (GLUT4) in muscle, and the prevention of decreased expression of GLUT4 in adipose tissue. Additionally, n3-LCPUFA has been reported to stimulate fatty acid oxidation in the liver via the activation of peroxisome proliferator activated receptor- $\alpha$ (PPAR- $\alpha$ ) [15]. Development of insulin resistance, which was previously thought to be a consequence of excess dietary intake in obesity and a consequence of genetic, environmental, dietary, and sedentary factors in diabetes mellitus may now be taken as a consequence of factors that are being released by the adipokinomes, and which include a number of proinflammatory cytokines.

Some of the adipokines secreted from the white adipose tissue (WAT) are dependent on TNF $\alpha$, which regulates synthesis of IL-6, acute phase protein, and haptoglobin [16]. Plasma levels of IL-6 reportedly increase during obesity [17], and IL-6 in addition to leptin, may convey information for regulation of energy balance from adipocytes to the hypothalamus. Circulating levels of IL-18 and IL-10 are also increased in obesity [18], and may play a role in the development of this disorder. The production of other cytokines including the ones mentioned has been attributed to hypoxia in areas of fat deposits with consequential increase in tissue mass with the increase in obesity. Hypoxia-inducible factor-1 (HIF-1), a transcriptional activation factor of leptin [19] may play a key role in the process.

\section{Some conventional medicines against obesity}

A number of approaches have been taken for anti-obesity drugs or drugs that can reduce obesity. A number of such approaches along with current and emerging drugs have been reviewed by Williams and his group [20]. The United States Food and Drug Administration (USFDA) has thus far approved five drugs, namely orlistat, phentermine/topiramate, lorcaserin, naltrexone/bupropion, and liraglutide to treat obesity. On the other hand, the European Medicines Agency (EMA) has approved only three orlistat, bupropion/naltrexone and liraglutide [21]. Orlistat is an inhibitor of pancreatic lipase thereby modulating the digestion and absorption of fat in the intestine. Side-effects of this drug include oily stools, diarrhea, abdominal pain, fecal spotting, There has also been a few reports of adverse effects on the hepatic system like cholelithiasis, cholostatic hepatitis, and subacute liver failure [22]. The combination therapy of phentermine and topiramate results in minor effects like insomnia and dry mouth, but also can produce serious side effects in the form of cardiovascular complications [23]. Mitral regurgitation is a serious side effect of lorcaserin, which may lead to other complications like increased risk of other cardiovascular complications [24]. Naltrexone is an opioid antagonist used for treatment of opioid and alcohol dependency, while buproprion is used for treating depression and help giving up smoking. Individually, the drugs have no or little effect on obesity; used in combination, they form a safe anti-obesity polypharmacy drug with no serious side effects except nausea [25]. Liraglutide, originally developed for treatment of diabetes, has now been approved as an anti-obesity drug. Adverse reactions of this drug include a number of gastrointestinal complaints like nausea, abdominal pain, decreased appetite, diarrhea, and constipation. Thyroid $\mathrm{C}$-cell tumors and acute pancreatitis are more severe effects that may arise from use of this drug [26].

Information sheet supplied by the National Institute of Diabetes and Digestive and Kidney diseases mention some more prescription drugs to treat obesity. These drugs include semaglutide, setmelanotide, benzphetamine, diethylpropion, and phendimetrazine. All these drugs have reported mild or serious adverse effects associated with their use [27]. Since anti-obesity drugs, despite their weight loss benefit can have adverse effects, and the only viable alternative to these drugs is bariatric surgery, scientists and traditional medicinal practitioners (TMPs) are taking a close look at the plant kingdom for plants that have the potential to reduce weight. Plants have the advantage of being easily available; an endangered plant can be cultivated to bring it out of its endangered status and increase availability. Moreover, any given plant can have hundreds of secondary metabolites, otherwise known as phytochemicals, with diverse pharmacological effects, one of which can be to reduce obesity.

\section{Anti-obesity plants and phytochemicals}

Several plants/plant parts, plant formulations, and phytochemicals have been described as anti-obesity plants [28]. These plants include Nigella sativa L. (Ranunculaceae, black cumin), Opuntia ficus-indica (L.) Miller (Cactaceae, prickly pear), Camellia sinensis (L.) Kuntze (Theaceae, tea), Crocus sativus L. (Iridaceae, crocus), Laminaria digitata (Hudson) J.V. Lamouroux (Laminariaceae, oarweed), and Lycium barbarum L. (Solanaceae, goji berry). Family and English names of the plants are given in parenthesis. Another review article gives the names of sixty-three antiobesity plants, including one Allium genus plant, namely Allium victorialis L. (Alliaceae, Alpine leek) [29]. Leaves of the plant (100 $\mathrm{mg} / \mathrm{kg}$ ) are consumed for two weeks to reduce weight. A very detailed review published in 2015 cites 118 plants/formulations, which are useful against obesity [30]. The review cites a number of useful Allium genus plants like Allium cepa L., Allium fistulosum L., Allium nigrum L., and Allium sativum L. Another review article on anti-obesity plants list 76 plants along with their geographic distribution [31]. This last review does not mention a single Allium genus plant as an anti-obesity plant. This is despite reports as to the efficacy of particularly Allium cepa and Allium sativum to reduce obesity.

The influence of quercetin-rich onion peel extracts have been examined on adipokine expression in visceral adipose tissue of rats in diet-induced obese rats. The extract influenced adipokine expressions, leading to moderation effect on obesity-induced inflammation [32]. Another study on the effect of quercetin-rich onion peel extract (OPE) supplementation to high fat-fed rats found that lipid accumulations and triglyceride concentrations in 3T3-L1 pre-adipocyte cells were suppressed; mRNA of activating protein-2 (AP-2) were down-regulated; mRNAs of carnitine palmitoyl transferase- $1 \alpha(\mathrm{CPT}-1 \alpha)$ and fatty acid binding protein 4 (FABP4) were up-regulated; retroperitoneal and mesenteric fat levels 
were lowered, which led the authors to conclude that quercetin-enriched OPE may inhibit obesity by "suppressing pre-adipocyte differentiation and inhibiting adipogenesis" [33]. A recent review on the effect of Allium sativum (garlic) on obesity mentions that garlic extract can reduce body weight, adipose tissue mass and improve lipid profiles in high fat diet fed rodents; ajoene and 1,2-vinyldithiin were among the active ingredients [34]. Because of the large number of papers already published on Allium cepa (1995 hits in PubMed Central with the search terms <Allium cepa, obesity >) and Allium sativum (3649 hits in PubMed Central with the search terms <Allium sativum, obesity>), we decided to concentrate on a lesser known species of Allium genus, namely Allium fistulosum and its effects on obesity.

\section{Allium fistulosum L. and its anti-obesity effects}

Allium fistulosum L. belongs to the Alliaceae/Amaryllidaceae family of plants and is known in English as Welsh onion, bunching onion or long green onion. The plant, unlike the common onion (Allium cepa) does not have a bulb and has hollow leaves. The plant is used in culinary dishes of China, Japan, and Korea. It is also used in culinary dishes of Russia and Jamaica.

Phytochemicals reported in Allium fistulosum include allicin, p-coumaric acid, ferulic acid, isoquercitrin, quercitrin, kaempferol, $\beta$-sitosterol, campesterol, and stigmasterol [35]. A cyclic monoterpene D-limonene has also been identified from the plant [36]. Alliin, rutin, and quercetin are three other phytochemicals reported from the plant [37].

A study conducted on male 8-week-old C57BL/6 J mice fed a high fat diet with or without aqueous extracts of Allium fistulosum demonstrated beneficial effects of the extracts on reducing obesity and several parameters related to obesity. The ethanolic and aqueous extract (AFE and AFW, respectively) containing ferulic acid and quercetin decreased body weight and furthermore, decreased adipose tissue and liver weight, and adipocyte size. Improvement in serum lipid profiles and adiponectin were also observed with AFE. Both extracts attenuated high fat dietinduced changes in serum leptin and insulin-like growth factor 1 levels, liver expression of 5'-adenosine monophosphate-activated protein kinase (AMPK), and adipose tissue expression of UCP2 (mitochondrial uncoupling protein 2) [38].

An herbal extract combining extracts of Allium fistulosum and Viola mandshurica W. Becker (Violaceae) to high-fat diet-induced obese mice resulted in reduced body and tissue weights, adipocyte size, lipid accumulation in the liver, and serum triglyceride, total cholesterol and leptin levels. The extracts also increased the mRNA expression of peroxisome proliferator-activated receptor- $\gamma$ (PPAR- $\gamma$ ), UCP2 and adiponectin and decreased expression of leptin in the epididymal white adipose tissue, resulting in reduction of obesity [39]. In this context it is to be noted that leptin is the gene product of the obesity gene, and plays a role in the regulation of human adipose tissue mass and body weight [40]. Adiponectin in combination with PPAR- $\gamma$ pl;ays a major role in the functioning of adipose tissue and ameliorating non-alcoholic fatty liver disease and the pathogenesis of obesity [41].

Ferulic acid, a phytoconstituent of Allium fistulosum, reportedly caused suppression in male C57BL/6N mice of high fat diet-induced weight gain and inhibited fatty acid biosynthesis [42]. Flavonols like quercetin (another phytoconstituent of Allium fistulosum) can inhibit adipogenesis by inhibiting uptake of methyl glucose in adipocytes [43], and induce apoptosis in mouse pre-adipocytes [44], or improve glucose uptake without adipogenesis activity in 3T3-L1 adipocytes [45]. Apoptosis was due to antioxidant activity of quercetin, which lead to down-regulation of PARP (poly (ADP-ribose) polymerase) and Bcl-2 proteins, and activation of caspase-3, Bax and Bak proteins. A phase 2 clinical trial is underway as to whether quercetin can change the absorption of glucose by the body in obese subjects [46]. There is indeed a high possibility of discovery of other flavonoid compounds playing a distinct role in reducing obesity.

Poor gut absorption and low bioavailability of flavonoids along with destruction of flavonoids during cooking are important factors raised by scientists in the use of flavonoid compounds to control obesity. Quercetin glycosides are generally more easily absorbed. The role of gut microbiota is another factor that needs to be examined in more detail. It is to be expected that administration of flavonoid compounds with specific side groups attached, use of probiotics to change gut microbiota composition, and finding out alternate routes of delivery of flavonoid compounds are the next phases of research that needs to be taken into consideration for control of obesity and obesity-induced disorders.

\section{Conclusion}

Obesity is a complicated disorder and which can lead to a number of noncommunicable diseases, including cancer, arthritis, and cardiovascular problems. Conventional medicines have moderate to serious side effects; plant-based medications like phytochemicals can offer a solution for reducing obesity. In this review, we show that Allium fistulosum and at least two of its reported phytochemicals, namely ferulic acid and quercetin can play a role in decreasing weight and obesity.

\section{Acknowledgement}

The study was funded totally by the authors.

\section{Conflicts of interest}

The authors declare no conflicts of interest.

\section{References}

1. World Health Organization (WHO). (2021) Obesity and overweight Factsheet.

2. Poirier P, Després J.-P. (2003) Obesity and cardiovascular disease. Medecine Sciences. 19(10): 943-949.

3. Parmar MY. (2018) Obesity and Type 2 diabetes mellitus. Integrative Obesity and Diabetes. 4(4): 1-2.

4. Kortt MA, Baldry J. (2002) The association between musculoskeletal disorders and obesity. Australian Health Review. 25(6): 207-214.

5. Basen-Engquist K, Chang M. (2011) Obesity and cancer risk: Recent review and evidence. Current Oncology Report. 13(1): 71-76.

6. Schmidt FM, Weschenfelder J, Sander C, Minkwitz J, Thormann J, Chittka T, Mergl R, Kirkby KC, Fa $\square$ hauer M, Stumvoll M, Holdt LM, Teupser D, Hegerl U, Himmerich H. (2015) Central obesity and modulating effects of physical activity. PLoS One. 10(3): e0121971.

7. Trayhurn P, Wood IS. (2004) Adipokines: inflammation and the pleiotropic role of white adipose tissue. British Journal of Nutrition. 92(3): 347-355.

8. Frühbeck G, Gómez-Ambrosi J, Muruzabal FJ \& Burrell MA. (2001) The adipocyte: a model for integration of endocrine and metabolic signaling in energy metabolism regulation. American Journal of Physiology-Endocrinology and Metabolism. 280(6): E827-E847.

9. Trayhurn P, Beattie JH. (2001) Physiological role of adipose tissue: white adipose tissue as an endocrine and secretory organ. Proceedings of the Nutrition Society. 60(3): 329-339.

10. Bulló M, Garcia-Lorda P, Megias I, Salas-Salvado J. (2003) Systemic inflammation, adipose tissue tumor necrosis factor, and leptin expression. Obesity Research. 11(4): 525-531.

11. Das UN. (2001) Is obesity an inflammatory condition? Nutrition. 17(11-12): 953-966. 
12. Hill JO. (2001) A new way of looking at obesity. Nutrition. 17(11-12): 975-976.

13. Dain A, Repossi G, Diaz-Gerevini GT, Vanamala J, Das UN, Eynard AR. (2016) Long chain polyunsaturated fatty acids (LCPUFAs) and nordihydroguaiaretic acid (NDGA) modulate metabolic and inflammatory markers in a spontaneous type 2 diabetes mellitus model (Stillman Salgado rats). Lipids in Health and Disease. 15: 205.

14. Das UN. (2002) Long-chain polyunsaturated fatty acids and diabetes mellitus. The American Journal of Clinical Nutrition. 75(4): 780-781.

15. Delarue J, LeFoll C, Corporeau C, Lucas D. (2004) n-3 long chain polyunsaturated fatty acids: a nutritional tool to prevent insulin resistance associated to type 2 diabetes and obesity? Reproduction Nutrition Development. 44(3): 289-299.

16. Chiellini C, Bertacca A, Novelli SE, Görgün CZ, Ciccarone A, Giordano A, Xu H, Soukas A, Costa M, Gandini D, Dimitri R, Bottone P, Cecchetti P, Pardini E, Perego L, Navalesi R, Folli F, Benzi L, Cinti S, Friedman JM, Hotamisligil GS, Maffei M. (2002) Obesity modulates the expression of haptoglobin in the white adipose tissue via TNF $\square$. Journal of Cellular Physiology. 190(2): 251-258.

17. Vozarova B, Weyer C, Hanson K, Tataranni PA, Bogardus C, Pratley RE. (2001) Circulating interleukin-6 in relation to adiposity, insulin action, and insulin secretion. Obesity Research. 9(7): 414-417.

18. Esposito K, Pontillo A, Ciotola M, Di Palo C, Grella E, Nicoletti G, Giugliano D. (2002) Weight loss reduces interleukin-18 levels in obese women. The Journal of Clinical Endocrinology \& Metabolism. 87(8): 3864-3866.

19. Ambrosini G, Nath AK, Sierra-Honigmann MR, FloresRiveros J. (2002) Transcriptional activation of the human leptin gene in response to hypoxia. Involvement of hypoxia-inducible factor 1. Journal of Biological Chemistry. 277(37): 3460134609.

20. Williams DM, Nawaz A, Evans M. (2020) Drug therapy in obesity: A review of current and emerging treatments. Diabetes Therapy. 11(6): 1199-1216.

21. Yumuk V, Tsigos C, Fried M, Schindler K, Busetto L, Micik D, Toplak H, Obesity Management Task Force of the European Association for the Study of Obesity. (2015) European Guideline for obesity management in adults. Obesity Facts. 8(6): 402-424.

22. Filippatos TD, Derdemezis CS, Gazi IF, Nakou ES, Mikhailidis DP, Elisaf MS. (2008) Orlistat-associated adverse effects and drug interactions. A critical review. Drug Safety. 31(1): 53-65.

23. Cosentino G, Conrad AO, Uwaifo GI. (2013) Phentermine and topiramate for the management of obesity: a review. Drug Design, Development and Therapy. 7: 267-278.

24. DiNicolantonio JJ, Chatterjee S, O'Keefe JH, Meier P. (2014) Lorcaserin for the treatment of obesity? A closer look at its side effects. Open Heart. 1(1): e000173.

25. Verpeut JL, Bello NT. (2014) Drug safety evaluation of naltrexone/buproprion for the treatment of obesity. Expert Opinion Drug Safety. 13(6): 831-841.

26. Onge E St., Miller SA, Motycka C. (2016) Liraglutide (Saxenda ${ }^{\circledR}$ ) as a Treatment for Obesity. Food and Nutrition Sciences. 7(4): 227-235.

27. National institute of Diabetes and Digestive and kidney Diseases Prescription Medications to Treat Overweight \& Obesity.

28. Hasani-Ranjbar S, Jouyandeh Z, Abdollahi M. (2013) A systematic review of anti-obesity medicinal plants - an update. Journal of Diabetes \& Metabolic Disorders. 12(1): 28.
29. Hussain SKS, Hakimullah, Aliuddin SM. (2019) Classical medicinal plants associated with anti obesity activity. International Journal of Pharmaceutical Sciences Review and Research. 54(1): 103-108.

30. Patra S, Nithya S, Srinithya B, Meenakshi SM. (2015) Review of medicinal plants for anti-obesity activity. 6(3): 21 .

31. de Freitas Junior LM, de Almeida Jr EB. (2017) Medicinal plants for the treatment of obesity: ethnopharmacological approach and chemical and biological studies. American Journal of Translational Research. 9(5): 2050-2064.

32. Kim OY, Lee S.-M, Do H, Moon J, Lee K.-H, Cha Y.-J, Shin M.-J. (2012) Influence of quercetin-rich onion peel extracts on adipokine expression in the visceral adipose tissue of rats. Phytotherapy Research. 26(3): 432-437.

33. Moon J, Do H.-J, Kim OH, Shin M.-J. (2013) Antiobesity effects of quercetin-rich onion peel extract on the differentiation of 3T3-L1 preadipocytes and the adipogenesis in high fat-fed rats. Food and Chemical Toxicology. 58: 347-354.

34. El-Saber Batiha G, Beshbishy AM, Wasef LG, Elewa YHA, AlSagan AA, El-Hack MEA, Taha AE, Abd-Elhakim YM, Devkota HP. (2020) Chemical constituents and pharmacological activities of garlic (Allium sativum L.): A review. Nutrients. 12: 872.

35. Vlase L, Parvu M, Parvu EA, Toiu A. (2013) Phytochemical analysis of Allium fistulosum L. and Allium ursinum L. Digest Journal of Nanomaterials and Biostructures. 8(1): 457-467.

36. Ajayi GO, Akinsanya MA, Agbabiyaka AT, Oyebanjo KS, Hungbo TD, Olagunju JA. (2019) D-Limonene: A major bioactive constituent in Allium fistulosum identified by GC-MS analysis. The Journal of Phytopharmacology. 8(5): 257-259.

37. Ţigu AB, Moldovan CS, Toma V.-A., Farcaş AD, Moţ AC, Jurj A, Fischer-Fodor E, Mircea C, Pârvu M (2021) Phytochemical analysis and in vitro effects of Allium fistulosum L. and Allium sativum L. extracts on human normal and tumor cell lines: A comparative study. Molecules. 26: 574.

38. Sung Y.-Y, Kim D.-S, Kim S.-H., Kim HK. (2018) Aqueous and ethanolic extracts of welsh onion, Allium fistulosum, attenuate high-fat diet-induced obesity. BMC Complementary and Alternative Medicine. 18: 105.

39. Sung Y.-Y, Kim S.-H, Yoo BW, Kim HK. (2015) The nutritional composition and anti-obesity effects of an herbal mixed extract containing Allium fistulosum and Viola mandshurica in high-fat-diet-induced obese mice. BMC Complementary and Alternative Medicine. 15: 370.

40. Maffei M, Halaas J, Ravussin E, Pratley RE, Lee GH, Zhang Y, Fei H, Kim S, Lallone R, Ranganathan S, Kern PA, Friedman JM. (1995) Leptin levels in human and rodent: Measurement of plasma leptin and ob RNA in obese and weight-reduced subjects. Nature Medicine. 1: 1155-1161.

41. Ishtiaq SM, Rashid H, Hussain Z, Arshad MI, Khan JA. (2019) Adiponectin and PPAR: a setup for intricate crosstalk between obesity and non-alcoholic fatty liver disease. Reviews in Endocrine and Metabolic Disorders. 20(3): 253-261.

42. Son MJ, Rico CW, Nam SH, Kang MY. (2010) Effect of oryzanol and ferulic acid on the glucose metabolism of mice fed with a high-fat diet. Journal of Food Science. 76(1): H7-H10.

43. Strobel P, Allard C, Perez-Acle T, Calderon R, Aldunate R, Leighton F. (2005) Myricetin, quercetin and catechin-gallate inhibit glucose uptake in isolated rat adipocytes. Biochemical Journal. 386(Pt 3): 471-478.

44. Hsu CL, Yen GC. (2006) Introduction of cell apoptosis in 3T3L1 pre-adipocytes by flavonoids is associated with antioxidant activity. Molecular Nutrition \& Food Research. 50(11): 10721079. 
45. Fang X.-K, Gao J, Zhu D.-N. (2008) Kaempferol and quercetin isolated from Euonymus alatus improve glucose uptake of 3T3L1 cells without adipogenesis activity. Life Science. 82(11-12): 615-622.
46. ClinicalTrials.gov. Investigating the use of quercetin on glucose absorption in obesity, and obesity with Type 2 diabetes. ClinicalTrials.gov Identifier: NCT00065676.
This work is licensed under Creative Commons Attribution 4.0 License

\section{To Submit Your Article Click Here: Submit Manuscript}

DOI: $10.31579 / 2640-1045 / 078$
Ready to submit your research? Choose Auctores and benefit from:

* fast, convenient online submission

* rigorous peer review by experienced research in your field

* rapid publication on acceptance

* authors retain copyrights

* unique DOI for all articles

* immediate, unrestricted online access

At Auctores, research is always in progress.

Learn more https://auctoresonline.org/journals/endocrinology-anddisorders 